


\title{
A GRID-INDEPENDENT APPROXIMATE RIEMANN SOLVER WITH APPLICATIONS TO THE EULER AND NAVIER-STOKES EQUATIONS
}

\author{
Christopher L. Rumsey ${ }^{\dagger}$ \\ NASA Langley Research Center, Hampton VA \\ Bram van Leer $\ddagger$ \\ Philip L. Roe $\ddagger$ \\ University of Michigan, Ann Arbor, MI
}

\begin{abstract}
A new two-dimensional approximate Riemann solver has been developed that obtains fuxes on grid faces via wave decomposition. By utilizing information propagating in the velocity-difference directions rather than in the grid-normal directions, this flux function more appropriately interprets and hence more sharply resolves shock and shear waves when they lie oblique to the grid. The model uses five waves to describe the difference in states at a grid face. Two acoustic waves, one shear wave, and one entropy wave propagate in the direction defined by the local velocity difference vector, while the fifth wave is a shear wave that propagates at a right angle to the other four. Test cases presented include a shock reflecting off a wall, a pure shear wave, supersonic flow over an airfoil, and viscous separated airfoil flow. Results using the new model give significantly sharper shock and shear contours than a grid-aligned solver. Navier-Stokes computations over an airfoil show reduced pressure distortions in the separated region as a result of the grid-independent upwinding.
\end{abstract}

\section{INTRODUCTION}

In one space dimension, upwind flow solvers have reached a highly sophisticated stage of development. Significant among these is the approximate Riemann solver of Roe ${ }^{1}$, a flux function that is derived by finding an exact solution to the system of Euler equations, linearized about an averaged state. The eigenvectors of the averaged flux Jacobian matrix represent acoustic, shear, and entropy waves that propagate in the grid direction with speeds equal to the corresponding eigenvalues. These waves describe the difference in states across each cell face and are used to determine the flux on each face.

Since most current multidimensional flow solvers that employ an approximate Riemann solver implement it by solving the one-dimensional problem in each grid direction separately, the full advantages of this method are lost. In reality, the waves can travel in infinitely many directions. Constraining them to the grid

\section{$\dagger$ Fluid Mechanics Division, Member AIAA \\ $\ddagger$ Professor, Member AIAA}

Copyright (C)1991 by the American Institute of Aeronautics and Astronautics, Inc. No copyright is asserted in the United States under Title 17, U.S. Code. The U.S. Government has a royalty-free license to exercise all rights under the copyright claimed herein for Governmental purposes. All other rights are reserved by the copyright owner directions is inconsistent with the physics of the situation and can result in improper interpretation of waves that are not aligned with the grid.

In recent years, several researchers have attempted to develop multidimensional upwind methods for the Euler equations. Davis ${ }^{2}$ uses the velocity field to determine the orientation of a hypothetical shock wave at each grid face and then determines flux components normal and tangential to this direction. This idea of a rotated Riemann solver has been further investigated by Levy et al. ${ }^{3}$ Levy uses the pressure gradient to construct the dominant-direction angle, then employs twodimensional interpolation of data from surrounding cells to determine left and right states both perpendicular and normal to this direction at each cell face. Two Roe-type Riemann solvers are then used in the rotated frames, and their results are combined to obtain the grid-direction flux.

Roe $^{4}$ has proposed a method in which the number and type of waves to represent the data are chosen in advance. Then angles and strengths of the waves are calculated using gradients in the data. For example, one type of decomposition consists of four acoustic waves, a shear wave, and an entropy wave. Eight unknowns need to be solved at each face.

A different method for upwinding in two dimensions is due to Hirsch et at and Deconinck et al. ${ }^{6}$ They employ a local diagonalization of the Euler equations based on two characteristic directions. This diagonalization can be exact, but is in general a "best" approximation in that directions are chosen to minimize off- 
diagonal terms. Two acoustic waves, one shear wave, and one entropy wave are used in the decomposition: the shear wave propagates parallel to the local pressure gradient and the acoustic waves propagate in the direction defined by the local strain rate tensor.

Powell and van Leer ${ }^{7}$ have developed a cell-vertex scheme based on downwind distribution. At the distribution step in the scheme, the cell-centered residuals are distributed to the surrounding nodes with weights determined by the local convection directions. This is similar to ideas developed by other researchers, including Giles et al. ${ }^{8}$ An excellent review of most of the multidimensional methods mentioned above is given by Powell. $^{9}$

The grid-independent approximate Riemann solver described in this paper uses five waves to describe the difference in states at a grid face. Two acoustic waves, a shear wave, and an entropy wave are assumed to propagate in a direction defined by the local velocity field, while a second shear wave propagates at a right angle to the other four waves. This paper first outlines the equations of motion and the finite volume formulation, then describes in detail the derivation of the 5-wave model. A stability analysis of an explicit time-stepping scheme is included, followed by numerical examples and conclusions.

\section{NOMENCLATURE}

A, B Jacobian matrices

a speed of sound

D̂ matrix defined by (40)

$E \quad$ specific total energy

$\mathbf{F}, \mathbf{G}, \mathbf{\Phi}$ inviscid fluxes

$\mathbf{F}_{\mathrm{v}}, \mathbf{G}_{\mathbf{v}}$ viscous fluxes

$H \quad$ specific total enthalpy

$k \quad$ heat transfer coefficient

M Mach number

$\mathbf{P}, \mathbf{R}$ eigenvectors

$p \quad$ pressure

$q \quad$ velocity in $\theta_{g}$ direction

$q^{\prime} \quad$ velocity in $\theta^{\prime}$ direction

Re Reynolds number

$r \quad$ velocity normal to $\theta_{g}$

$r^{\prime} \quad$ velocity normal to $\theta^{\prime}$

$s \quad$ length along cell face

$T$ temperature

$t$ time

U conserved variables

$u, v \quad$ velocity components in $x$ and $y$ directions

$V \quad$ cell volume
$\vec{V}$

$\mathbf{W}$

$w$

$\boldsymbol{x}, \boldsymbol{y}$

$\alpha$

$\beta$

$\Gamma$

$\gamma$

$\Delta$

$\delta$

$\delta_{i j}$

$\zeta$

$\eta$

$\theta$

$\theta^{\prime}$

$\lambda$

$\mu$

$\nu$

$\rho$

$\tau_{i j}$

$\boldsymbol{\Omega}$

$\omega$

Subscripts:

$d \quad$ velocity-difference direction

$f \quad$ flow direction

$g \quad$ grid direction

$i, j \quad$ grid indices

$L, R \quad$ from the left, right

Superscripts:

$\begin{array}{ll}\wedge & \begin{array}{l}\text { Roe-averaged } \\ \text { nondimensional } \\ \sim\end{array} \\ -\quad \text { cell-averaged }\end{array}$

TWO-DIMENSIONAL EQUATIONS

The two-dimensional Navier-Stokes equations can be written as

$$
\frac{\partial \mathbf{U}}{\partial t}+\frac{\partial \mathbf{F}}{\partial x}+\frac{\partial \mathbf{G}}{\partial y}=\frac{\partial \mathbf{F}_{\mathbf{v}}}{\partial x}+\frac{\partial \mathbf{G}_{\mathbf{v}}}{\partial y}
$$

where the conserved variables are $\mathbf{U}=[\rho, \rho u, \rho v, \rho E]^{T}$ and the inviscid flux vectors are

$$
\mathbf{F}=\left[\begin{array}{c}
\rho u \\
\rho u^{2}+p \\
\rho u v \\
\rho u H
\end{array}\right] \quad \mathbf{G}=\left[\begin{array}{c}
\rho v \\
\rho u v \\
\rho v^{2}+p \\
\rho v H
\end{array}\right]
$$


The viscous fluxes axe

$$
\mathbf{F}_{\mathbf{v}}=\left[\begin{array}{c}
0 \\
\tau_{11} \\
\tau_{21} \\
u_{j} \tau_{1 j}+k \frac{\partial T}{\partial x}
\end{array}\right] \quad \mathbf{G}_{\mathrm{v}}=\left[\begin{array}{c}
0 \\
\tau_{12} \\
\tau_{22} \\
u_{j} \tau_{2 j}+k \frac{\partial T}{\partial y}
\end{array}\right],
$$

where

$$
\tau_{i j}=\mu\left(\frac{\partial u_{i}}{\partial x_{j}}+\frac{\partial u_{j}}{\partial x_{i}}\right)+\lambda \frac{\partial u_{k}}{\partial x_{k}} \delta_{i j} .
$$

The ideal gas equation-of-state closes the set of equations:

$$
p=(\gamma-1) \rho\left[E-\frac{u^{2}+v^{2}}{2}\right] .
$$

Since the flux function developed in this paper is only applied to the inviscid fluxes (viscous terms are always centrally differenced), the viscous terms are ignored throughout the remainder of the derivation.

The resultant Euler equations can be written in quasilinear form:

$$
\frac{\partial \mathbf{W}}{\partial t}+\mathbf{A} \frac{\partial \mathbf{W}}{\partial x}+\mathbf{B} \frac{\partial \mathbf{W}}{\partial y}=\mathbf{0},
$$

where $W$ is the vector of primitive variables, $W=$ $[\rho, u, v, p]^{T}$, and $\mathrm{A}$ and $\mathrm{B}$ are the Jacobian matrices

$$
\begin{aligned}
& \mathbf{A}=\left[\begin{array}{cccc}
u & \rho & 0 & 0 \\
0 & u & 0 & 1 / \rho \\
0 & 0 & u & 0 \\
0 & \rho a^{2} & 0 & u
\end{array}\right] \\
& \mathbf{B}=\left[\begin{array}{cccc}
v & 0 & \rho & 0 \\
0 & v & 0 & 0 \\
0 & 0 & v & 1 / \rho \\
0 & 0 & \rho a^{2} & v
\end{array}\right] .
\end{aligned}
$$

Traveling wave solutions to (6) are of the form

$$
\mathbf{W}(x, y, t)=\mathbf{W}(x \cos \theta+y \sin \theta-\lambda t)
$$

where $\theta$ is the angle that defines the direction of wave propagation. Substitution of (9) into (6) results in the eigenvalue problem

$$
(\mathbf{A} \cos \theta+\mathbf{B} \sin \theta) \delta \mathbf{W}=\lambda \delta \mathbf{W},
$$

where $\delta \mathbf{W}$ is the amplitude of the traveling wave. The four eigenvalues and corresponding right eigenvectors of (10) are:

$$
\begin{aligned}
& \lambda_{1}=u \cos \theta+v \sin \theta+a \\
& \lambda_{2}=u \cos \theta+v \sin \theta-a \\
& \lambda_{3}=u \cos \theta+v \sin \theta \\
& \lambda_{4}=u \cos \theta+v \sin \theta
\end{aligned}
$$

$$
\begin{aligned}
& \mathbf{P}_{1}=\left[1, \stackrel{a}{\rho} \cos \theta, \frac{a}{\rho} \sin \theta, a^{2}\right]^{T} \\
& \mathbf{P}_{2}=\left[1,-\frac{a}{\rho} \cos \theta,-\frac{a}{\rho} \sin \theta, a^{2}\right]^{T} \\
& \mathbf{P}_{3}=\left[0,-\frac{a}{\rho} \sin \theta, \frac{a}{\rho} \cos \theta, 0\right]^{T} \\
& \mathbf{P}_{4}=[1,0,0,0]^{T} .
\end{aligned}
$$

These eigenvectors represent: (1) a positive acoustic disturbance, (2) a negative acoustic disturbance, (3) a shear wave, and (4) an entropy wave. The eigenvalues represent their corresponding propagation speeds.

\section{FINITE VOLUME FORMULATION}

The finite volume form of the Euler equations can be written as

$$
\frac{\partial \overline{\mathbf{U}}_{i, j}}{\partial t}=-\frac{1}{V_{i, j}}\left[\sum_{\ell=1}^{m}(\mathbf{F} \Delta y-\mathbf{G} \Delta x)_{\ell}\right]_{i, j},
$$

where $\overline{\mathrm{U}}_{i, j}$ represents the vector of cell-averaged conserved quantities for the $(i, j)$ th grid cell, $V_{i, j}$ is the area of cell $(i, j)$, and $(\Delta x)_{\ell}$ and $(\Delta y)_{\ell}$ are the changes of $x$ and $y$ along the $\ell$ th cell face. For a quadrilateral grid, $m=4$. The cell face length is defined as

$$
\Delta s=\sqrt{\Delta x^{2}+\Delta y^{2}}
$$

and the outward velocity normal to the cell face is

$$
\begin{aligned}
q & =(u \Delta y-v \Delta x) / \Delta s \\
& =u \cos \theta_{g}+v \sin \theta_{g},
\end{aligned}
$$

while a velocity parallel to the grid face is given by

$$
\begin{aligned}
r & =(u \Delta x+v \Delta y) / \Delta s \\
& =-u \sin \theta_{g}+v \cos \theta_{g} .
\end{aligned}
$$

With these definitions, equation (13) can be written

$$
\frac{\partial \overline{\mathbf{U}}_{i, j}}{\partial t}=-\frac{1}{V_{i, j}}\left[\sum_{\ell=1}^{m} \Phi_{\ell} \Delta s_{\ell}\right]_{i, j},
$$

where the flux vector $\Phi$ is the flux normal to each face of the control volume and is given by

$$
\mathbf{\Phi}=\left[\begin{array}{c}
\rho q \\
\rho q u+p \cos \theta_{g} \\
\rho q v+p \sin \theta_{g} \\
\rho q H
\end{array}\right] .
$$

Numerically, the fluxes on the faces are determined through the use of a flux function

$$
\mathbf{\Phi}_{k+1 / 2}=\mathbf{\Phi}\left[\left(\mathbf{W}_{L}\right)_{k+1 / 2},\left(\mathbf{W}_{R}\right)_{k+1 / 2}\right],
$$

where $k$ represents the grid index $i$ or $j$. The primitive variables are obtained on the cell faces via one-point 
extrapolation for first-order spatial differencing and via standard MUSCL-type ${ }^{10}$ extrapolation for higher order. The subscripts $L$ and $R$ denote the directional bias of the extrapolation. The exact form of the numerical flux function (19) is discussed in the next section.

\section{FLUX FUNCTIONS}

\section{A. Grid-Aligned Approximate Riemann Solver}

The grid-aligned flux function of Roe $^{1}$ is briefly outlined in this section. The flux at a face is

$$
\boldsymbol{\Phi}=\frac{1}{2}\left(\Phi_{\mathbf{L}}+\mathbf{\Phi}_{\mathbf{R}}\right)-\frac{1}{2} \sum_{k=1}^{4}\left|\hat{\lambda}_{k}\right| \Omega_{k} \hat{\mathbf{R}}_{k} .
$$

The eigenvectors are given by

$$
\begin{aligned}
& \hat{\mathbf{R}}_{1}=\left[1, \hat{u}+\hat{a} \cos \theta_{g}, \hat{v}+\hat{a} \sin \theta_{g}, \hat{H}+\hat{a} \hat{q}\right]^{T} \\
& \hat{\mathbf{R}}_{2}=\left[1, \hat{u}-\hat{a} \cos \theta_{g}, \hat{v}-\hat{a} \sin \theta_{g}, \hat{H}-\hat{a} \hat{q}\right]^{T} \\
& \hat{\mathbf{R}}_{3}=\left[0,-\hat{a} \sin \theta_{g}, \hat{a} \cos \theta_{g}, \hat{a} \hat{r}\right]^{T} \\
& \hat{\mathbf{R}}_{4}=\left[1, \hat{u}, \hat{v}, \frac{1}{2}\left(\hat{u}^{2}+\hat{v}^{2}\right)\right]^{T}
\end{aligned}
$$

for the equations written in conserved variable form. The $k$ th wave of this system has a strength $\Omega_{k}$, evaluated as the $k$ th component of the vector $\boldsymbol{\Omega}$, where

$$
\mathbf{\Omega}=\left[\begin{array}{c}
\frac{1}{2 \hat{q}^{2}}(\Delta p+\hat{\rho} \hat{a} \Delta q) \\
\frac{1}{2 \hat{a}^{2}}(\Delta p-\hat{\rho} \hat{a} \Delta q) \\
\frac{1}{\hat{a}^{2}}\left(\hat{a}^{2} \Delta \rho-\Delta p\right)
\end{array}\right]
$$

and $\Delta(\cdot)=(\cdot)_{R}-(\cdot)_{L}$. The wavespeeds are

$$
\begin{aligned}
& \hat{\lambda}_{1}=\hat{q}+\hat{a} \\
& \hat{\lambda}_{2}=\hat{q}-\hat{a} \\
& \hat{\lambda}_{3}=\hat{q} \\
& \hat{\lambda}_{4}=\hat{q} .
\end{aligned}
$$

The Roe-averaged values (denoted by hats) are defined as

$$
\begin{aligned}
\hat{\rho} & =\sqrt{ }_{\rho_{L}} \rho_{R} \\
\hat{u} & =u_{L} w+u_{R}(1-w) \\
\hat{v} & =v_{L} w+v_{R}(1-w) \\
\hat{H} & =H_{L} w+H_{R}(1-w) \\
\hat{a} & =\sqrt{(\gamma-1)\left[\hat{H}-\frac{1}{2}\left(\hat{u}^{2}+\hat{v}^{2}\right)\right],}
\end{aligned}
$$

where $w=\sqrt{ } \rho_{L} /\left(\sqrt{ } \rho_{L}+\sqrt{ } \rho_{R}\right)$ and $\hat{q}$ and $\hat{r}$ are defined by (15) and (16), using $\hat{u}$ and $\hat{v}$.

One can interpret this model in a geometric sense by looking at the effects of the acoustic and shear waves in $(\Delta u, \Delta v, \Delta p)$-space, as shown in figure 1 . (The entropy wave only causes a change in the density, so it is not representable in this space.) $L$, the representation of the state to the left of the cell face, is placed at the origin and the right state $\mathrm{R}$ is located at $(\Delta u, \Delta v, \Delta p)$, as determined by the differences between $\mathrm{L}$ and $\mathrm{R}$. All waves propagate in the $\theta_{g}$ direction, represented by the vertical plane in the figure. The acoustic and shear waves have the following properties:

$$
\begin{array}{cl}
\text { +acoustic : } & \frac{\delta p}{\rho a^{2}}=\frac{\delta u}{a \cos \theta_{g}}=\frac{\delta v}{a \sin \theta_{g}} \\
\text {-acoustic : } & \frac{\delta p}{\rho a^{2}}=-\frac{\delta u}{a \cos \theta_{g}}=-\frac{\delta v}{a \sin \theta_{g}} \\
\text { shear : } & -\frac{\delta u}{a \sin \theta_{g}}=\frac{\delta v}{a \cos \theta_{g}} .
\end{array}
$$

Hence, in addition to the entropy wave, the difference in states is decomposed into: (1) a +acoustic wave in the $\theta_{g}$ direction, which gives a positive change in pressure along with a positive change in velocity along $\theta_{\eta}$, (2) a -acoustic wave in the $\theta_{q}$ direction, which gives a positive change in pressure along with a negative change in velocity along $\theta_{g}$, and (3) a shear wave in the $\theta_{g}$ direction, which gives no change in pressure and a change in velocity normal to $\theta_{g}$. These waves are sketched in figure 1 .

\section{B. Grid-Independent Approximate Riemann Solver}

The motivation belind the development of the present grid-independent Riemann solver is the desire to be able to recognize and appropriately model both shock and shear waves regardless of their orientation with respect to the grid. The method for accomplishing this goal is the following: (1) choose a direction of wave propagation more physically appropriate than the directions defined by the grid, (2) represent the difference in $L$ and $R$ states with a combination of acoustic, shear, and entropy waves, and (3) form a flux in the grid direction from the information propagating in the physically-relevant grid-independent direction.

Step 1. The wave propagation direction chosen for step 1 is the velocity difference direction

$$
\theta_{d}=\tan ^{-1}\left(\frac{\Delta v}{\Delta u}\right)
$$

defined from $-\frac{\pi}{2}$ to $\frac{\pi}{2}$. In this direction, the differences between the two states can be interpreted either as a compression normal to $\theta_{d}$ or a shear aligned with $j_{d}$. Figure 2 is a graplical representation of the angle $\theta_{d}$.

Step 2. In this step, the difference in states is modeled by a combination of two acoustic waves and an entropy wave propagating in the $\theta_{d}$ direction, as well as a shear wave propagating in the $\left(\theta_{d}+\frac{\pi}{2}\right)$ direction. This shear wave causes a change in velocity parallel to $\theta_{d}$ with no change in pressure, so it allows for sharp capturing 
of oblique shear waves in a computation. The representation in primitive variable form of this $\left(\theta_{d}+\frac{\pi}{2}\right)$ shear wave is

$$
\begin{aligned}
\mathbf{P}_{\left(\theta_{d}+\pi / 2\right) \text { shear }} & =\left[\begin{array}{c}
0 \\
-\frac{a}{\rho} \sin \left(\theta_{d}+\frac{\pi}{2}\right) \\
\frac{a}{\rho} \cos \left(\theta_{d}+\frac{\pi}{2}\right) \\
0
\end{array}\right] \\
& =\left[\begin{array}{c}
0 \\
-\frac{a}{\rho} \cos \theta_{d} \\
-\frac{a}{\rho} \sin \theta_{d} \\
0
\end{array}\right] .
\end{aligned}
$$

The two acoustic waves and the entropy wave are represented by $\mathbf{P}_{1}, \mathbf{P}_{2}$, and $\mathbf{P}_{4}$ in (12), with $\theta=\theta_{d}$.

There is not a unique way to determine the combination of these waves such that

$$
\Delta \mathrm{W}=\sum_{k=1}^{4} \Omega_{k} \hat{\mathbf{P}}_{k}
$$

is satisfied. The entropy wave is always present with a strength given by $\Omega_{4}$ in (22), but there is some freedom in picking the strengths of the other three. One strategy is to choose them such that the pathlength in $(\Delta u, \Delta v, \Delta p)$-space is minimized. This minimum pathlength model is accomplished by using either two acoustic waves and an entropy wave or one acoustic, a $\left(\theta_{d}+\frac{\pi}{2}\right)$ shear, and an entropy wave. (Recall that the entropy wave is not representable in $(\Delta u, \Delta v, \Delta p)$-space.) The choice depends on the location in phase space of the right state $R$ relative to the cone defined by all acoustic waves emanating from $\mathrm{L}$. By definition, $\mathrm{R}$ lies in the $\theta_{d}$ plane. If $R$ resides inside the "acoustic cone," as is the case with $R_{1}$ in figure $3(a)$, then two acoustic waves describe the shortest path. If $R$ resides outside the cone, as represented by $R_{2}$ in the figure, then one acoustic and $a\left(\theta_{d}+\frac{\pi}{2}\right)$ shear wave describe the shortest path. The mathematical conditions for $R$ inside or outside the acoustic cone are:

$$
\begin{array}{cl}
\text { Inside : } & (\Delta p)^{2} \geq\left[\hat{\rho} \hat{a}\left(\Delta u \cos \theta_{d}+\Delta v \sin \theta_{d}\right)\right]^{2} \\
\text { Outside : } & (\Delta p)^{2}<\left[\hat{\rho} \hat{a}\left(\Delta u \cos \theta_{d}+\Delta v \sin \theta_{d}\right)\right]^{2}
\end{array}
$$

A second strategy is to choose the strengths of the acoustic and shear waves such that the path is in some sense closest to the straight line connecting $L$ and $R$ in phase space. More specifically, the area between the waves (taken in a certain order) and the direct path $L$ $\mathbf{R}$ is minimized. This minimum area model is due to Parpia. ${ }^{11}$ A geometric representation is given in figure 3(b), where again the entropy wave, although present, is not pictured. If $R$ lies inside the acoustic cone, like $R_{1}$ in the figure, then the path that minimizes the area (shaded region) is accomplished by two acoustic waves. If $R$ lies outside the cone, as represented by $R_{2}$, then some combination of two acoustic waves and a $\left(\theta_{d}+\frac{\pi}{2}\right)$ shear wave gives the minimum area. The exact expression will be given below.

Numerical experiments indicate that the minimum area model tends to give somewhat better results than the minimum pathlength model for a wide range of test cases, so it is used for all the computations in this paper. Numerical experiments also show that these models can produce nonlinear feedback that results in oscillatory flowfields and nonconvergence. The problem arises as a result of small changes in the computed values of $\theta_{\boldsymbol{d}}$ which feed back into the solution, thus producing further changes in $\theta_{d}$. An easy way to inhibit this feedback is to freeze the computed values of $\theta_{d}$ at each face at some point in the computation, calling them $\theta_{d}^{\prime}$. This freezing process results in the requirement of at least one additional wave to describe the differences between the left and right states at each face, since the state $R$ does not necessarily lie in the $\theta_{d}^{\prime}$ plane. A shear wave propagating in the $\theta_{d}^{\prime}$ direction produces a change in velocity normal to $\theta_{d}^{\prime}$, and can therefore be used as the additional wave.

Step 3. Finally, the flux on each grid face is calculated. The combination of the four waves from (28) plus the $\theta_{d}^{\prime}$ shear wave from freezing $\theta_{d}$ results in a 5 -wave model, which can be written as a family of schemes with a free parameter $\beta$. This family includes both the minimum pathlength and minimum area models discussed above. The flux is written as

$$
\mathbf{\Phi}=\frac{1}{2}\left(\Phi_{\mathbf{L}}+\Phi_{\mathbf{R}}\right)-\frac{1}{2} \sum_{k=1}^{5}\left|\hat{\lambda}_{k}\right| \mathbf{\Omega}_{k} \hat{\mathbf{R}}_{k}
$$

where the five waves are given by

$$
\begin{aligned}
& \hat{\mathbf{R}}_{1}=\left[1, \hat{u}+\hat{a} \cos \theta_{d}^{\prime}, \hat{v}+\hat{a} \sin \theta_{d}^{\prime}, \hat{H}+\hat{a} \hat{q}^{\prime}\right]^{T} \\
& \hat{\mathbf{R}}_{2}=\left[1, \hat{u}-\hat{a} \cos \theta_{d}^{\prime}, \hat{v}-\hat{a} \sin \theta_{d}^{\prime}, \hat{H}-\hat{a} \hat{q}^{\prime}\right]^{T} \\
& \hat{\mathbf{R}}_{3}=\left[0,-\hat{a} \cos \theta_{d}^{\prime},-\hat{a} \sin \theta_{d}^{\prime},-\hat{a} \hat{q}^{\prime}\right]^{T} \\
& \hat{\mathbf{R}}_{4}=\left[0,-\hat{a} \sin \theta_{d}^{\prime}, \hat{a} \cos \theta_{d}^{\prime}, \hat{a} \hat{r}^{\prime}\right]^{T} \\
& \hat{\mathbf{R}}_{5}=\left[1, \hat{u}, \hat{v}, \frac{1}{2}\left(\hat{u}^{2}+\hat{v}^{2}\right)\right]^{T} .
\end{aligned}
$$

These represent, respectively: $+\theta_{d}^{\prime}$ acoustic, $-\theta_{d}^{\prime}$ acoustic, $\left(\theta_{d}^{\prime}+\frac{\pi}{2}\right)$ shear, $\theta_{d}^{\prime}$ shear, and $\theta_{d}^{\prime}$ entropy waves. Also,

$$
\begin{aligned}
& \hat{q}^{\prime}=\hat{u} \cos \theta_{d}^{\prime}+\hat{v} \sin \theta_{d}^{\prime} \\
& \hat{r}^{\prime}=-\hat{u} \sin \theta_{d}^{\prime}+\hat{v} \cos \theta_{d}^{\prime},
\end{aligned}
$$

and hatted values are defined by (24). The wavestrengths are defined as

$$
\boldsymbol{\Omega}=\left[\begin{array}{c}
\frac{\Delta p}{2 \hat{a}^{2}}+\beta \frac{\hat{\rho}}{2 \hat{\hat{a}}}\left(\Delta u \cos \theta_{d}^{\prime}+\Delta v \sin \theta_{d}^{\prime}\right) \\
\frac{\Delta p}{2 \hat{a}^{2}}-\beta \frac{\hat{\rho}}{2 \hat{\hat{a}}}\left(\Delta u \cos \theta_{d}^{\prime}+\Delta v \sin \theta_{d}^{\prime}\right) \\
(\beta-1) \hat{\hat{e}}\left(\Delta u \cos \theta_{d}^{\prime}+\Delta v \sin \theta_{d}^{\prime}\right) \\
\frac{\hat{\rho}}{\hat{a}}\left(-\Delta u \sin \theta_{d}^{\prime}+\Delta v \cos \theta_{d}^{\prime}\right) \\
\frac{1}{\hat{a}^{2}}\left(\hat{a}^{2} \Delta \rho-\Delta p\right)
\end{array}\right] .
$$


The minimum pathlength model is obtained when $\beta$ is taken as

$$
\beta=\min \left[\left|\frac{\Delta p /(\hat{\rho} \hat{a})}{\Delta u \cos \theta_{d}^{\prime}+\Delta v \sin \theta_{d}^{\prime}}\right|, 1\right] .
$$

It can also be shown that the minimum area model, used for all computations in this paper, results when

$$
\beta=\min \left[\left\{\frac{\Delta p /(\hat{\rho} \hat{a})}{\left|\Delta u \cos \theta_{d}^{\prime}\right|+\left|\Delta v \sin \theta_{d}^{\prime}\right|}\right\}^{2}, 1\right] .
$$

In practice, a small number is added to the denominators in (35) and (36) to avoid division by zero in regions of null gradient. Also, $\beta$ is generally limited to be greater than 0.05 and is frozen along with $\theta_{d}^{\prime}$ as an aid to convergence.

The wavespeed associated with each of the waves in (32) is the average flowspeed in the direction of wave propagation, plus or minus the average speed of sound for the acoustic waves. Since the flux (31) is in the grid direction, however, it is necessary to take the components of these wavespeeds in the $\theta_{g}$ direction. They are:

$$
\begin{aligned}
& \hat{\lambda}_{1}=\left(\hat{q}^{\prime}+\hat{a}\right) \cos \left(\theta_{d}^{\prime}-\theta_{g}\right) \\
& \hat{\lambda}_{2}=\left(\hat{q}^{\prime}-\hat{a}\right) \cos \left(\theta_{d}^{\prime}-\theta_{g}\right) \\
& \hat{\lambda}_{3}=\hat{r}^{\prime}\left\{-\sin \left(\theta_{d}^{\prime}-\theta_{g}\right)\right\} \\
& \hat{\lambda}_{4}=\hat{q}^{\prime} \cos \left(\theta_{d}^{\prime}-\theta_{g}\right) \\
& \hat{\lambda}_{5}=\hat{q}^{\prime} \cos \left(\theta_{d}^{\prime}-\theta_{g}\right) .
\end{aligned}
$$

Notice that this 5 -wave model reduces to the gridaligned approximate Riemann solver when $\beta=1$ and $\theta_{d}^{\prime}=\theta_{g}$.

\section{TIME INTEGRATION AND STABILITY ANALYSIS}

The time integration and stability analysis of an explicit time-marching scheme with the 5 -wave model are discussed in this section. The stability analysis requires the eigenvalues of the Fourier transform of the right-hand side of

$$
\Delta t \underset{\partial t}{\partial \overline{\mathbf{U}}_{i, j}}=-\frac{\Delta t}{\bar{V}_{i, j}}\left[\sum_{\ell=1}^{m}(\mathbf{F} \Delta y-\mathbf{G} \Delta x)_{\ell}\right]_{i, j} .
$$

For first-order spatial differencing, this Fourier transform is the matrix

$$
\begin{gathered}
\Gamma\left(\zeta^{(i)}, \zeta^{(j)}\right)= \\
\nu \\
-\left\{\omega\left(\theta_{g}^{(i)}\right)+\omega\left(\theta_{g}^{(j)}\right)\right\} \\
i^{\partial \hat{\Phi}\left(\theta_{g}^{(i)}\right)} \sin \zeta^{(i)}+\hat{\mathbf{D}}\left(\theta_{g}^{(i)}\right)\left(1-\cos \zeta^{(i)}\right)+\left(1-\cos \zeta^{(j)}\right) \\
\partial \mathbf{U} \\
\left.+i \frac{\partial \hat{\Phi}\left(\theta_{g}^{(i)}\right)}{\partial \mathbf{U}} \sin \zeta^{(i)}\right]
\end{gathered}
$$

where $\theta_{g}^{(i)}$ and $\theta_{g}^{(j)}$ are the directions normal to the grid faces in the $i$ and $j$ directions, respectively, and $D$ is the matrix that satisfies

$$
\hat{\mathbf{D}} \Delta \mathbf{U}=\sum_{k=1}^{5}\left|\hat{\lambda}_{k}\right| \boldsymbol{\Omega}_{k} \hat{\mathbf{R}}_{k}
$$

The variable $\nu$ is the CFL number, defined as

$$
\nu=\left\{\omega\left(\theta_{g}^{(i)}\right)+\omega\left(\theta_{g}^{(j)}\right)\right\} \frac{\Delta t}{V_{i, j}},
$$

and $\omega\left(\theta_{g}^{(i)}\right)$ and $\omega\left(\theta_{g}^{(j)}\right)$ are the maximum wave speeds $|q|+a$ in each of the grid directions. For this analysis, everything is linearized about the cell-centers, and the grid faces are assumed to be of unit length. The parameters $\zeta^{(i)}$ and $\zeta^{(j)}$ are the wavelengths of the perturbations in the $i$ and $j$ directions, respectively.

For stability, the locus of the eigenvalues of the Fourier transform, often called the "Fourier footprint," must lie inside the stability boundary of the marching scheme. In general, the Fourier footprint of the 5-wave model is a function of $\rho, \nu, \mathbf{M}, \theta_{f}, \beta, \theta_{d}^{\prime}$, and $\theta_{g}$, as well as the perturbation wavelengths. The density is taken as the freestream value. Since the Fourier footprint is a function of so many variables, it is difficult to perform a thorough numerical analysis. However, an extensive number of variations in the independent variables have been tested. Based on the results obtained the following trends are noted. (1) The relative magnitudes of the eigenvalues are strongly dependent on the Mach number. In general, increasing $M$ increases the magnitudes, but at larger and larger $M$ an asymptotic limit is reached. (2) At low to moderate Mach numbers, $\beta$ has a significant effect on the eigenvalues, while at very high Mach numbers $\beta$ has very little effect. (3) Many of the modes of the Fourier footprint can have eigenvalues that lie on the imaginary axis. In particular, this occurs when $\theta_{f}$ and $\theta_{d}^{\prime}$ differ by $90^{\circ}$.

From this analysis, it is clear that the explicit forward-Euler time stepping scheme would be unstable for many of the modes, since the stability boundary for forward-Euler is a unit circle centered at $(-1,0)$ and does not include any part of the imaginary axis except the origin. However, 2-stage or higher schemes can be designed which satisfy this requirement. For example, the 4-stage scheme.

$$
\begin{aligned}
\mathbf{U}^{*} & =\mathbf{U}^{n}+\eta \Delta t \operatorname{Res}\left(\mathbf{U}^{n}\right) \\
\mathbf{U}^{* *} & =\mathbf{U}^{n}+\frac{1}{3} \Delta t \operatorname{Res}\left(\mathbf{U}^{*}\right) \\
\mathbf{U}^{* * *} & =\mathbf{U}^{n}+\frac{1}{2} \Delta t \operatorname{Res}\left(\mathbf{U}^{* *}\right) \\
\mathbf{U}^{n+1} & =\mathbf{U}^{n}+\Delta t \operatorname{Res}\left(\mathbf{U}^{* * *}\right)
\end{aligned}
$$


has a stability region including a finite part of the imaginary axis whenever $\eta<0.6756$.

An attempt was made to devise a "worst case" Fourier footprint for this scheme by choosing independent variables that yield the largest eigenvalue extent in the Real-Imaginary plane. This was accomplished by choosing $\mathrm{M}=100, \theta_{f}=90^{\circ}, \beta=0, \theta_{g}=0^{\circ}$, and $\theta_{d}^{\prime}=22.5^{\circ}$. Given this footprint, an $\eta$ of about 0.15 is "optimum" for the 4-stage scheme in the sense that it allows for the largest $\nu$ for stability. A plot of the Fourier footprint at its maximum $\nu=1.75$, along with the corresponding time stepping stability boundary using $\eta=0.15$ is shown in figure 4 . In the present paper, this 4-stage scheme is used for all Euler computations. Note that since the Fourier footprint is highly dependent on Mach number, the maximum allowable $\nu$ is actually higher than 1.75 for $M$ lower than 100. For example, at $M=3$ the maximum CFL number is 2.2, while at $M=1$ it is 2.5 , according to this linearized stability analysis.

The 5-wave model has also been incorporated into an implicit approximate factorization scheme, CFL2D, ${ }^{12}$ which is used for the Navier-Stokes computations in this paper. The stability analysis of the implicit scheme is not given here. However, it has been found in practice to converge for $\nu$ less than 4 with first-order spatial differencing and less than 2 with second-order differencing for both Euler and Navier-Stokes when the left-hand side flux Jacobians are approximated by:

$$
\begin{aligned}
& \left(\frac{\partial \Phi}{\partial \mathbf{U}}\right)_{L, k+\frac{1}{2}}=\frac{1}{2}\left[\left(\frac{\partial \Phi}{\partial \mathbf{U}}\right)_{k}+\left|\frac{\partial \hat{\Phi}}{\partial \mathbf{U}}\right|_{k+\frac{1}{2}}\right] \\
& \left(\frac{\partial \Phi}{\partial \mathbf{U}}\right)_{R, k+\frac{1}{2}}=\frac{1}{2}\left[\left(\frac{\partial \Phi}{\partial \mathbf{U}}\right)_{k+1}-\left|\frac{\partial \hat{\Phi}}{\partial \mathbf{U}}\right|_{k+\frac{1}{2}}\right]
\end{aligned}
$$

where $k$ represents the grid index $i$ or $j$.

\section{RESULTS}

\section{A. Shock Reflection}

The $M=2.9$ inviscid shock reflection case is computed on a $49 \times 17$ Cartesian mesh 4.8 units wide by 1.6 units high. An oblique shock enters the domain from the upper left corner, reflects off the bottom wall, and exits out the right end. The flow is turned through an angle of $10^{\circ}$. The nondimensional boundary conditions (nondimensionalized by combinations of $\rho_{\infty}$ and $\left.a_{\infty}\right)$ are: at inflow $\tilde{\rho}=1, \tilde{\rho} \tilde{u}=2.9, \tilde{\rho} \tilde{v}=0$, and $\tilde{\rho} \tilde{E}=5.9907$; at the top boundary $\tilde{\rho}=1.6328$, $\tilde{\rho} \tilde{u}=4.3272, \tilde{\rho} \tilde{v}=-0.7630$, and $\tilde{\rho} \tilde{E}=9.5091 ;$ at the back boundary, outflow conditions are extrapolated via second-order extrapolation from the interior; at the body, simple reflection boundary conditions are used.
Computations are initiated from freestream conditions and are run until the $\ell_{2}$-norm of the residual of all four equations drops below $1 \times 10^{-12}$. A first-order computation using the grid-aligned solver, run at a $\nu$ of 2.2 , converges in 158 iterations using about $2.53 \mathrm{CPU}$ seconds on the Cray 2 computer. Figure $5(a)$ shows pressure contours, nondimensionalized by $p_{\infty}$, while figure 5 (b) shows nondimensional pressure values along three $j=$ constant cuts through the mesh. Figures $6(a)$ and (b) show first-order results using the 5-wave model. Again, $\nu$ is taken as 2.2. $\theta_{d}$ is computed every iteration for the first 20 iterations, then only once every 20 iterations until the $\log$ of the $\ell_{2}$-norm of the residual drops to below -3.5 . After this, $\theta_{d}$ remains frozen. This 5wave model solution converges in 245 iterations, using about $4.80 \mathrm{CPU}$ seconds. It yields much sharper shocks than the grid-aligned method, although there are oscillations in the computed flowfield as well. The removal of these oscillations will be the subject of a forthcoming paper. ${ }^{13} \mathrm{~A}$ comparison of the residual histories is given in figure 7.

\section{B. Oblique Shear Wave}

The oblique shear wave case is computed on a $61 \times$ 21 Cartesian mesh 3 units wide by 1 unit high. Fluid enters the domain from the lower face at a $45^{\circ}$ angle and exits out the top face. To the right of the shear wave $M=1.51$, while to the left $M=1.812$. There is one transition cell where $M=1.661$. The nondimensional boundary conditions are: to the left of the shear $\tilde{\rho}=$ $1, \tilde{\rho} \tilde{u}=1.282, \tilde{\rho} \tilde{v}=1.282$, and $\tilde{\rho} \tilde{E}=3.427$; to the right of the shear $\tilde{\rho}=1, \tilde{\rho} \tilde{u}=1.068, \tilde{\rho} \tilde{v}=1.068$, and $\tilde{\rho} \tilde{E}=2.926$; at the transition zone $\tilde{\rho}=1, \tilde{\rho} \tilde{u}=1.175$, $\tilde{\rho} \tilde{v}=1.175$, and $\tilde{\rho} \tilde{E}=3.165 ;$ on the top and right boundaries, outflow conditions are extrapolated from the cells $45^{\circ}$ upstream.

A first-order Euler computation using the gridaligned method smears the shear wave significantly, as shown in figures $8(\mathrm{a})$ and (b). The 5-wave model, using the same strategy for freezing $\theta_{d}$ as for the shock reflection case, converges to the results shown in figures $9(a)$ and (b). The Mach contours are now very sharp, although oscillations are also present near the shear wave.

\section{Supersonic Flow About an Airfoil}

An Euler computation is performed for the NACA 0012 airfoil at $M=1.2, \alpha=0^{\circ}$ on a $65 \times 410$-mesh with an outer boundary extent of 15 chords and an average minimum spacing of 0.005 chords. Simple reflection boundary conditions are applied at the airfoil surface and freestream conditions are prescribed in ghost cells at the outer boundary. At these conditions, the NACA 0012 airfoil has a flowfield with a curved bow shock located in front of the airfoil and oblique shocks emanating from the trailing edge. 
Figures $10(a)$ and (b) show pressure contours for first-order computations using the grid-aligned model and the 5-wave model, respectively. (The 5-wave model result was obtained by restarting the grid-aligned solution with $\theta_{d}$ frozen the entire time. 2021 iterations beyond the restart are required to converge the $\ell_{2}$-norm of the residual to $1 \times 10^{-12}$ at a $\nu$ of 2.2.) The 5 -wave model gives significantly sharper shocks than the gridaligned method. Second-order results, using $\nu=1.1$, are shown in figures 11 (a) and (b). Again, the 5-wave model resolves the shocks better, although it shows little difference with the results of the first-order 5-wave model on this grid.

\section{Viscous Flow About an Airfoil With Separation}

Figure 12(a) shows pressure contours over a NACA 0012 airfoil at $\mathrm{M}=0.5, \alpha=3^{\circ}, \mathrm{Re}=5000$ using CFL2D with the grid-aligned scheme and second-order spatial accuracy on a $129 \times 49 \mathrm{C}$-mesh. The grid has an outer boundary extent of 14 chords and an average minimum spacing at the body of 0.0004 chords. This test case is taken from Venkatakrishnan. ${ }^{14}$ The results show a distorted pressure field over the rear half of the airfoil upper surface. Venkatakrishnan suggests a possible explanation for this numerical phenomenon: after flow separation, the detached shear layer is not oriented with the grid. Hence the grid-aligned solver misinterprets shear as a combination of shear and compression, resulting in a distortion of the computed pressure within the shear layer.

When the 5-wave model is employed, the distortions in the pressure contours essentially disappear, as shown in figure 12(b). Here, the model was restarted from the grid-aligned solution with $\theta_{d}$ frozen and $\nu=$ 2. Both the grid-aligned and 5 -wave model solutions are converged to $1 \times 10^{-6}$. It should be noted that for Navier-Stokes solutions the 5 -wave model can give nearly zero dissipation on grid faces aligned with the flow in the boundary layer, resulting in odd-even point decoupling there. This decoupling is alleviated by offsetting $\theta_{d}^{\prime}$ by a small amount whenever $\theta_{d}^{\prime} \approx \theta_{f} \approx \theta_{g} \pm \frac{\pi}{2}$.

\section{CONCLUSIONS}

A new two-dimensional approximate Riemann solver has been developed that obtains fluxes on grid faces via wave decomposition. By utilizing information propagating in the velocity difference directions rather than in the grid normal directions, this flux function more appropriately interprets and hence more sharply resolves shock and shear waves when they lie oblique to the grid. Five waves are used to model the difference in states at the faces. Four of these propagate in the velocity-difference direction and one, a shear wave, propagates $90^{\circ}$ to this.
Some modes of the Fourier footprint of this scheme possess eigenvalues that lie on the imaginary axis, so it is necessary that the stability boundary of an explicit time-stepping scheme includes a finite portion of the imaginary axis. A 4-stage scheme has been chosen to satisfy this requirement. It is stable up to a CFL number of at least 1.75 .

Discontinuities that lie oblique to the grid are resolved significantly better using the 5 -wave model than using a grid-aligned model, although the results can show oscillations in the flowfield. Computations of viscous separated flow over an airfoil indicate reduced pressure distortions in the separated region as a result of the grid-independent upwinding.

\section{REFERENCES}

1. Roe, P., "Approximate Riemann Solvers, Parameter Vectors, and Difference Schemes," JCP, 43, 1981, pp. 357-372.

2. Davis, S., "A Rotationally Biased Upwind Difference Scheme for the Euler Equations," NASA CR 172179, 1983; also JCP, 56, 1984, pp. 65-92.

3. Levy, D., Powell, K., Van Leer, B., "An Implementation of a Grid-Independent Upwind Scheme for the Euler Equations," AIAA 89-1931-CP, 1989.

4. Roe, P., "Discrete Models for the Numerical Analysis of Time-Dependent Multidimensional Gas Dynamics," NASA CR 172574, 1985; also JCP, 63, 1986, pp. 458-476.

5. Hirsch, Ch., Lacor, C., Deconinck, H., "Convection Algorithms Based on a Diagonalization Procedure for the Multidimensional Euler Equations," AIAA 87-1163-CP, 1987.

6. Deconinck, H., Hirsch, Ch., Peuteman, J., "Characteristic Decomposition Methods for the Multidimensional Euler Equations," 10th International Conference on Numerical Methods in Fluid Dynamics, Beijing, 1986.

7. Powell, K., Van Leer, B., "A Genuinely MultiDimensional Upwind Cell-Vertex Scheme for the Euler Equations," AIAA 89-0095, 1989.

8. Giles, M., Anderson, W., Roberts, T., "The Upwind Control-Volume Scheme for Unstructured Triangular Grids," NASA TM 101664, 1989.

9. Powell, K., "Towards a Genuinely Multi-Dimensional Upwind Scheme," presented at CFD Lecture Series at VKI, 1990.

10. Van Leer, B., "Upwind-Difference Methods for Aerodynamic Problems Governed by the Euler Equations," Lectures in Applied Mathematics, Vol. 22, 1985, pp. 327-336.

11. Parpia, I., "A Planar Oblique Wave Model for the Euler Equations," extended abstract submitted to the AIAA 10th Computational Fluid Dynamics Conference, Honolulu, Hawaii, 1991. 
12. Rumsey, C., "Details of the Computed Flowfield Over a Circular Cylinder at Reynolds Number 1200," Journal of Fluids Engineering, Vol. 110, 1988, pp. 446-452.

13. Rumsey, C., Van Leer, B., Roe, P., "Effect of a Multi-Dimensional Flux Function on the Monotonicity of Euler and Navier-Stokes Computa-

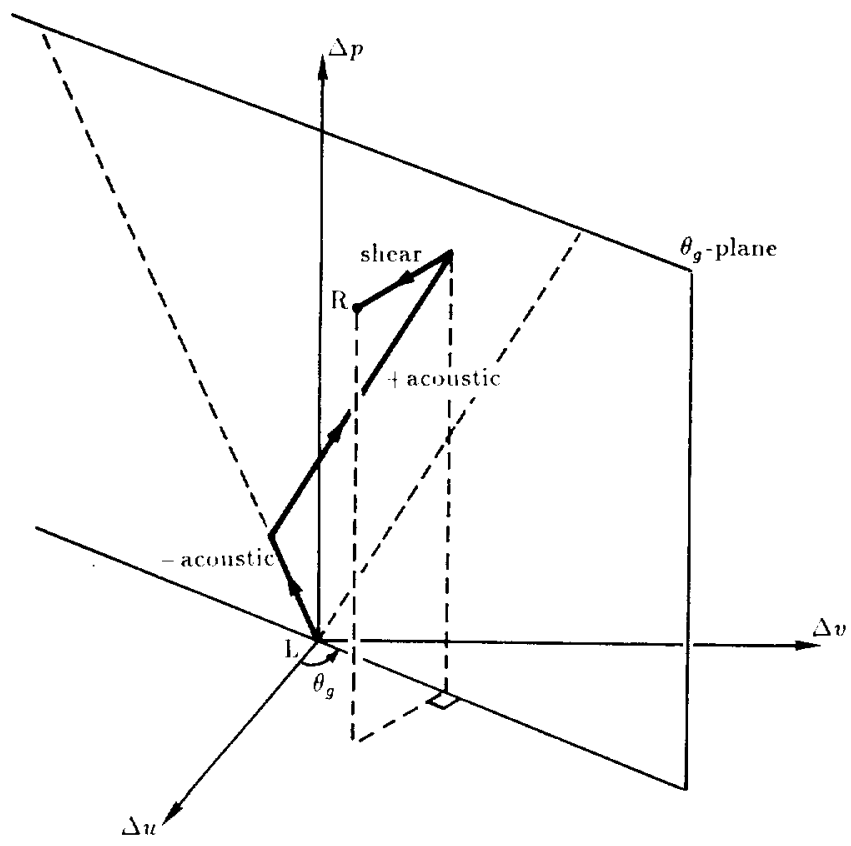

Figure 1 Geometric interpretation of grid-aligned wave model; entropy wave not shown

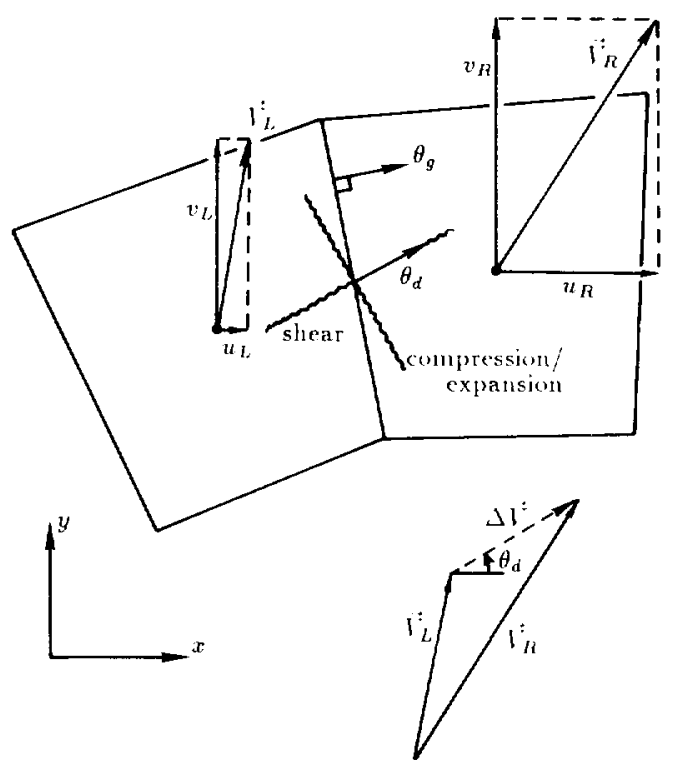

Figure 2 Graphical representation of $\theta_{d}$ tions," extended abstract submitted to the AIAA 10th Computational Fluid Dynamics Conference, Honolulu, Hawaii, 1991.

14. Venkatakrishnan, V., "Viscous Computations Using a Direct Solver," Computers and Fluids, Vol. 18, No. 2, 1990, pp. 191-204.
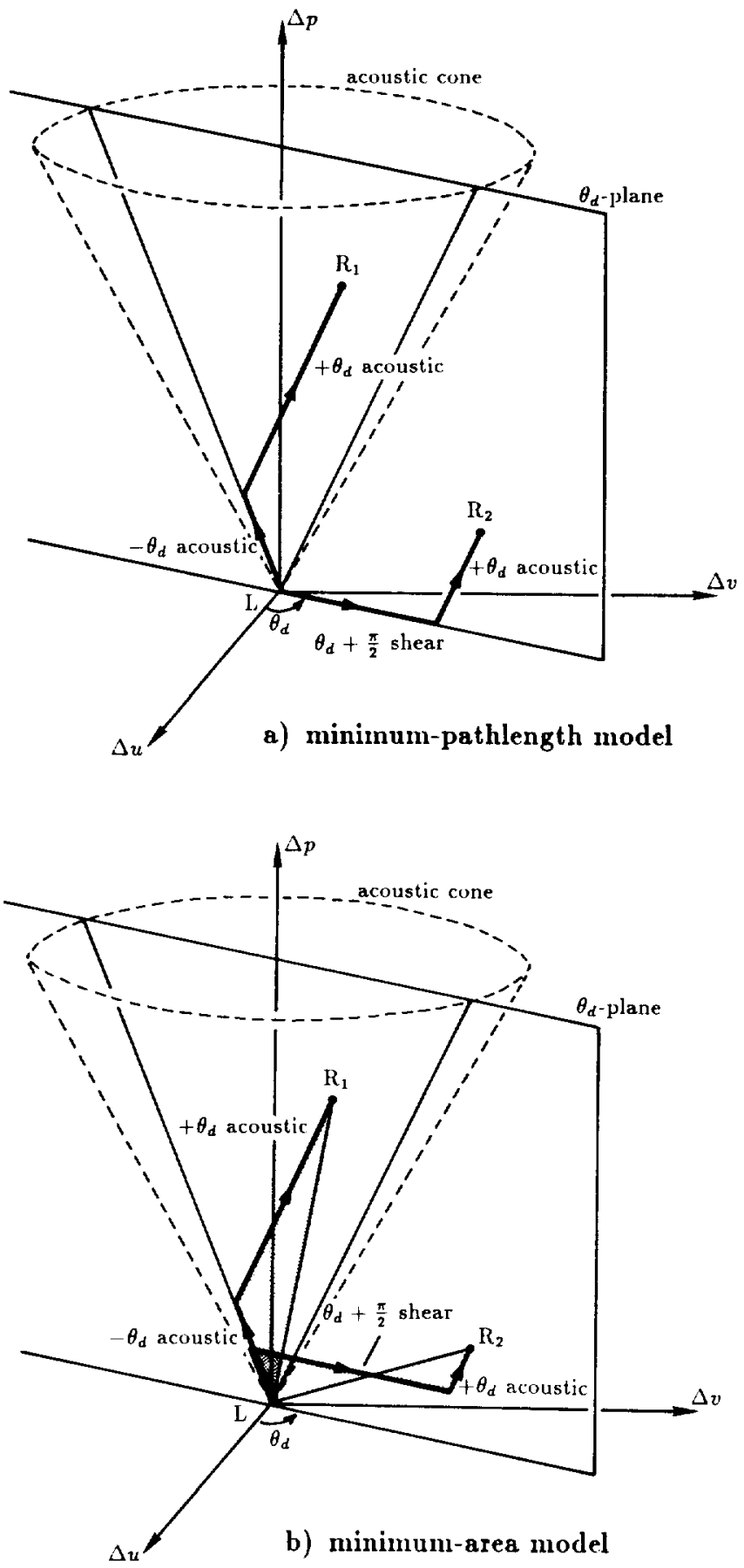

Figure 3 Geometric interpretation of grid-independent wave models 


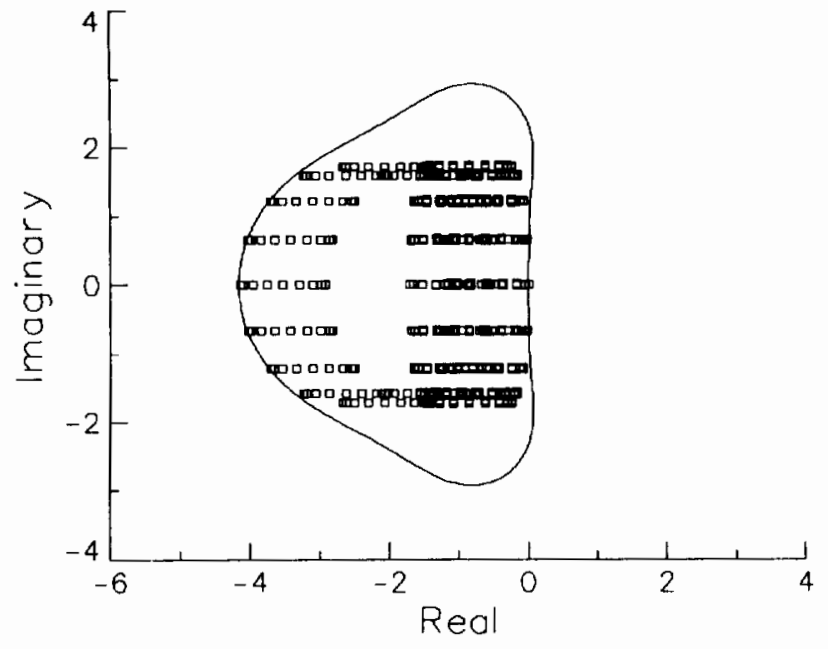

Figure 4 Stability boundary of 4-stage time-marching scheme with $\eta=0.15$, and "worst case" Fourier footprint of spatial differencing scheme for $\nu=$ 1.75

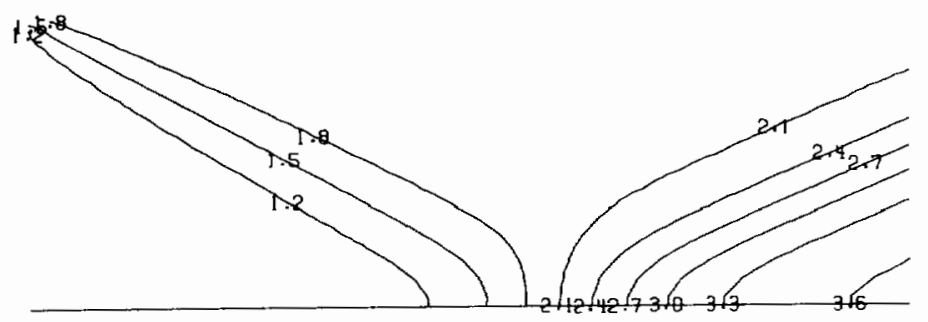

a) pressure contours

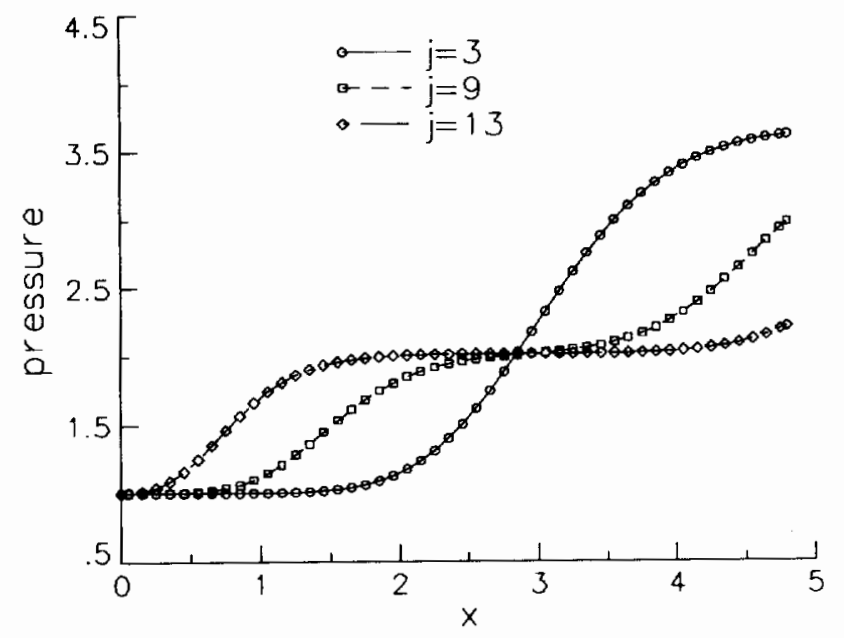

b) pressure along $j=$ constant lines

Figure 5 Shock reflection, grid-aligned wave model, 1st order accuracy

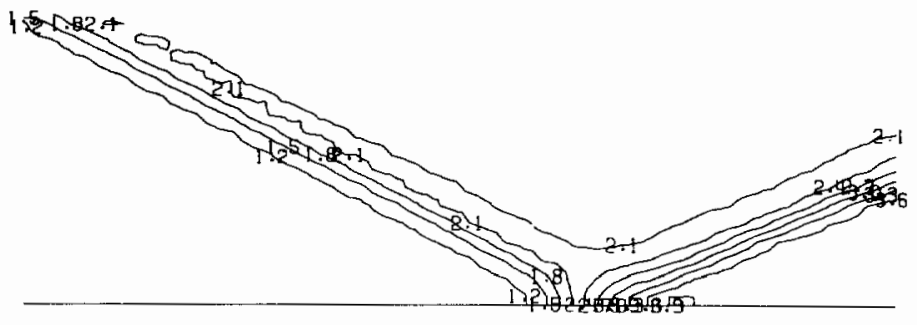

a) pressure contours

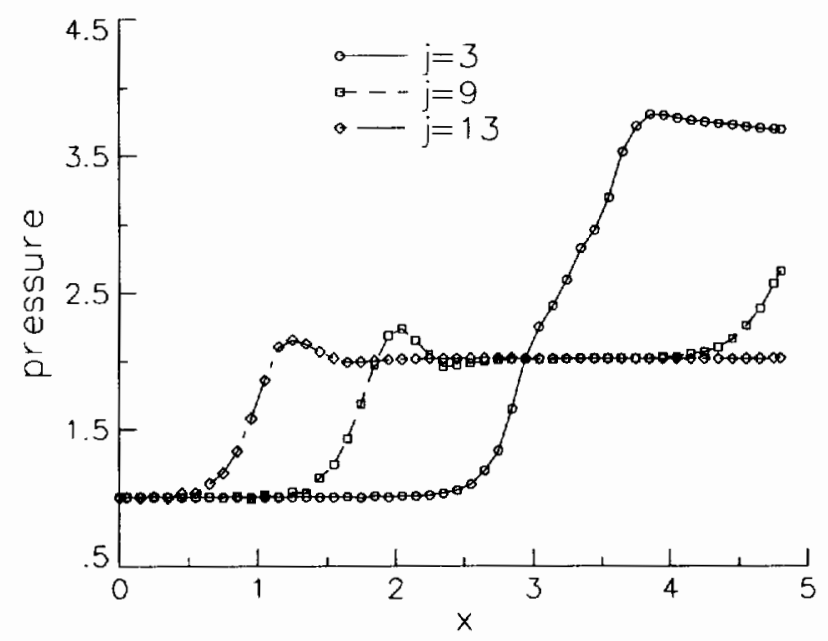

b) pressure along $j=$ constant lines

Figure 6 Shock reflection, 5-wave model, 1st order accuracy

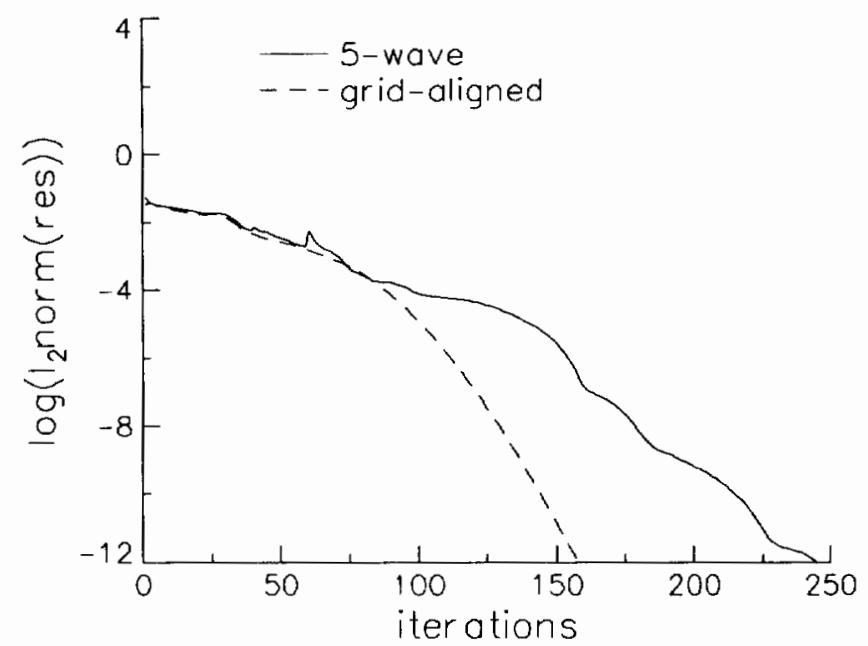

Figure 7 Shock reflection residual histories, 1st order accuracy 


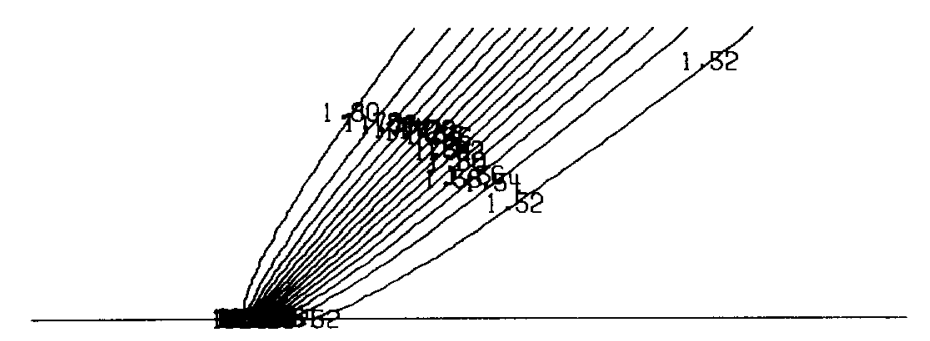

a) Mach contours

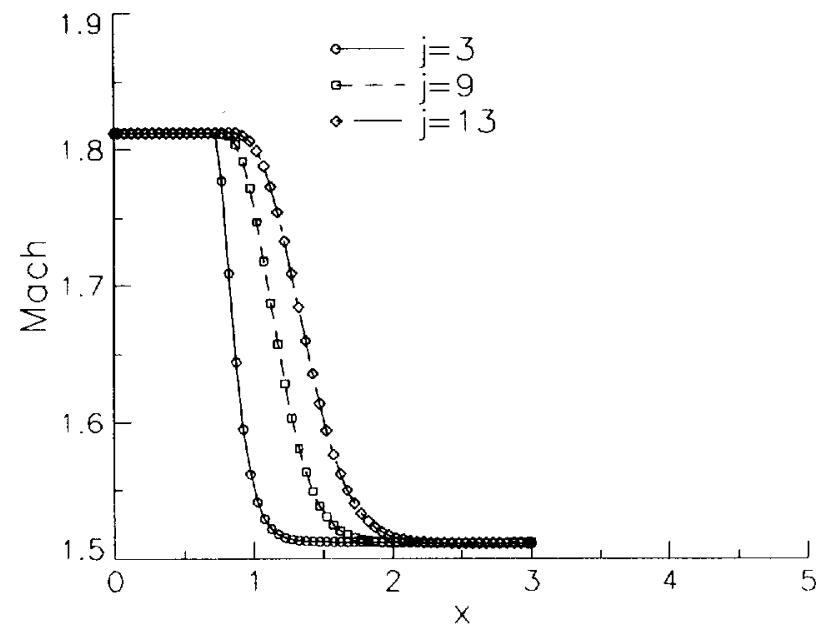

b) Mach number along $j=$ constant lines

Figure 8 Oblique shear wave, grid-aligned wave model, 1st order accuracy

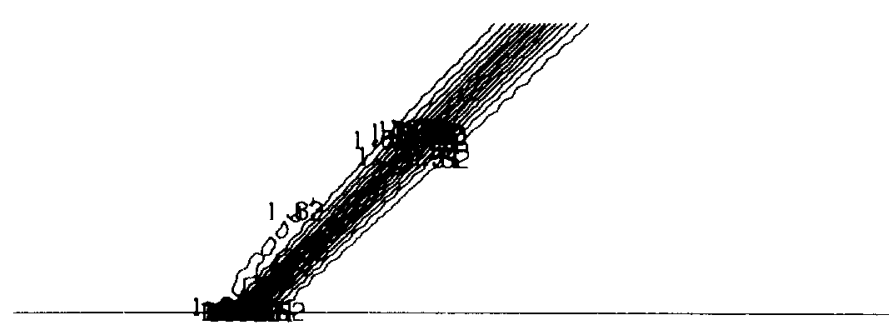

a) Mach contours

Figure 9 Oblique shear wave, 5-wave model, 1st order accuracy

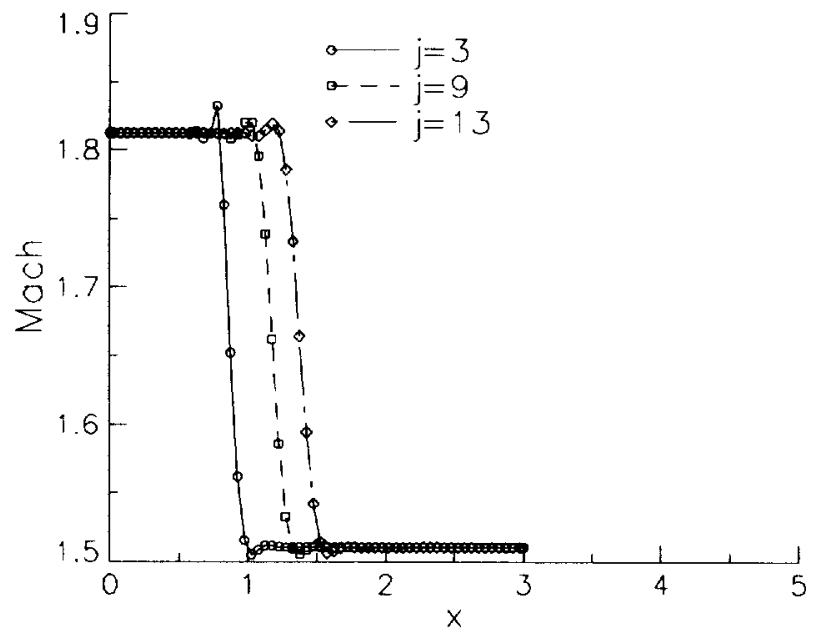

b) Mach number along $j=$ constant lines

Figure 9 Continued

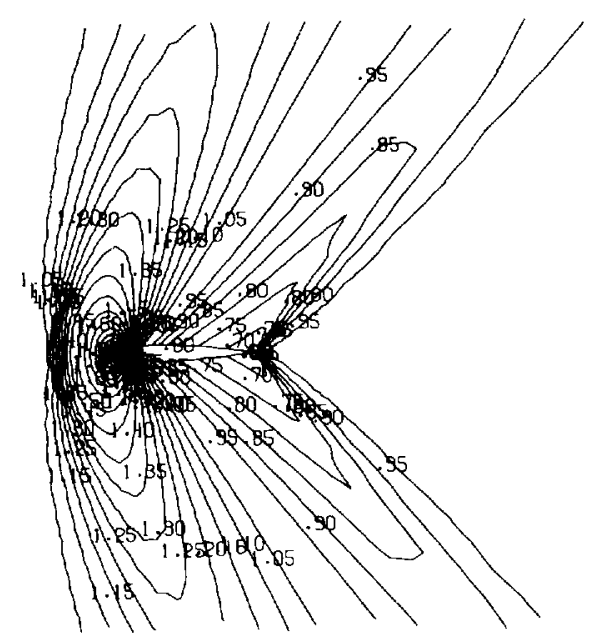

a) grid-aligned wave model

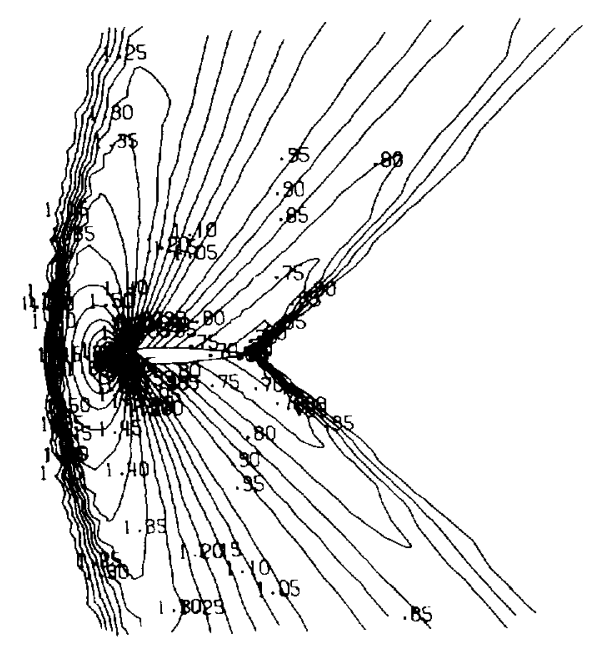

b) 5-wave model

Figure 10 NACA 0012, $\mathrm{M}=1.2, \alpha=0^{\circ}$, pressure contours, 1st order accuracy 


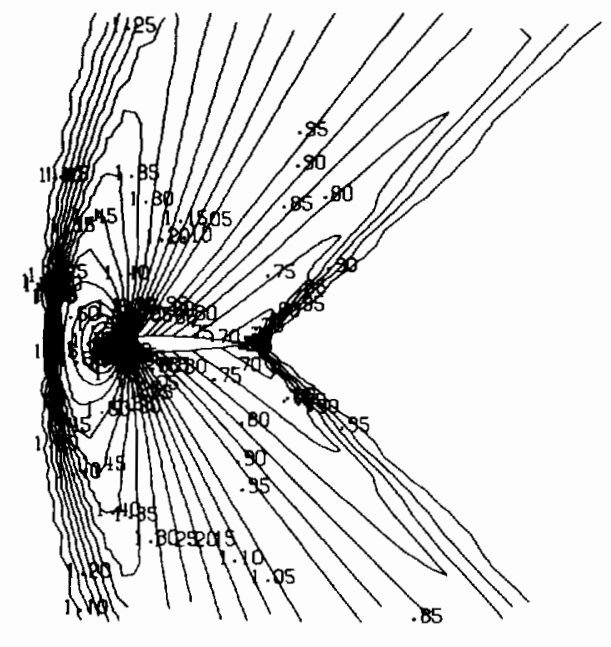

a) grid-aligned wave model

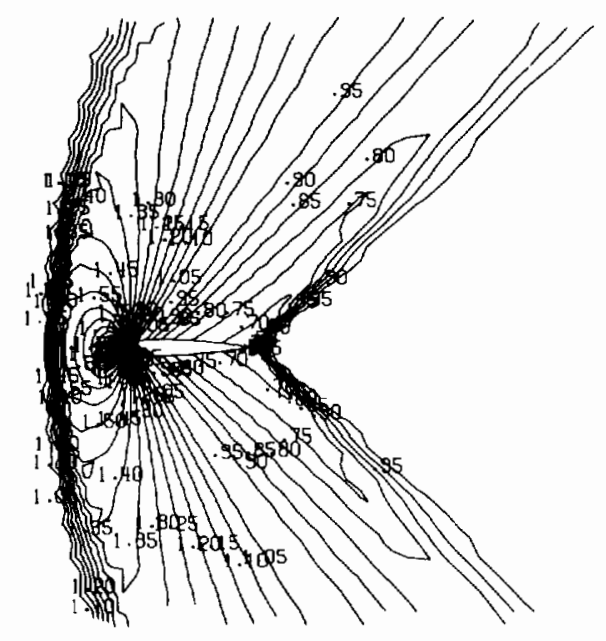

b) 5-wave model

Figure 11 NACA 0012, $\mathrm{M}=1.2, \alpha=0^{\circ}$, pressure contours, 2nd order accuracy

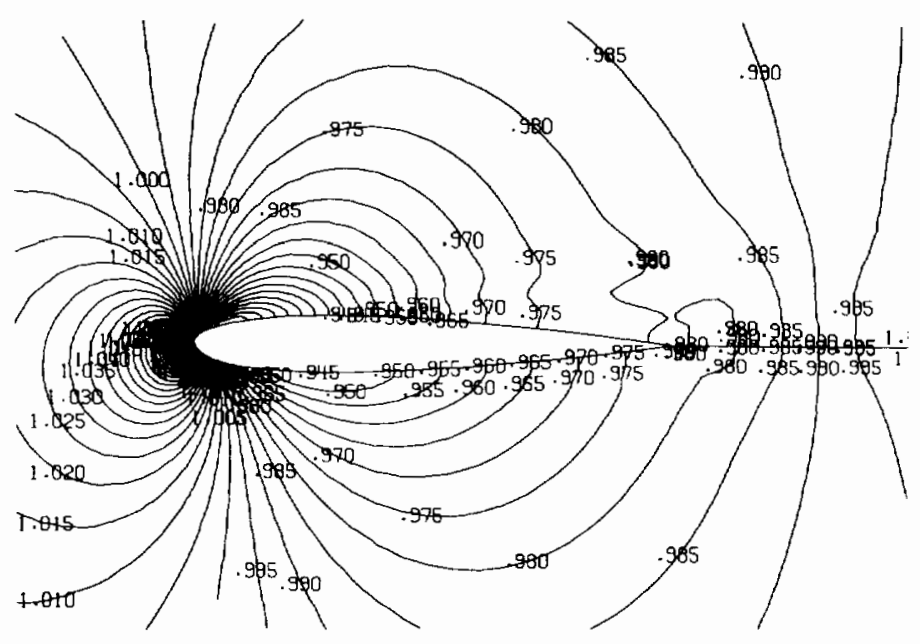

a) grid-aligned wave model

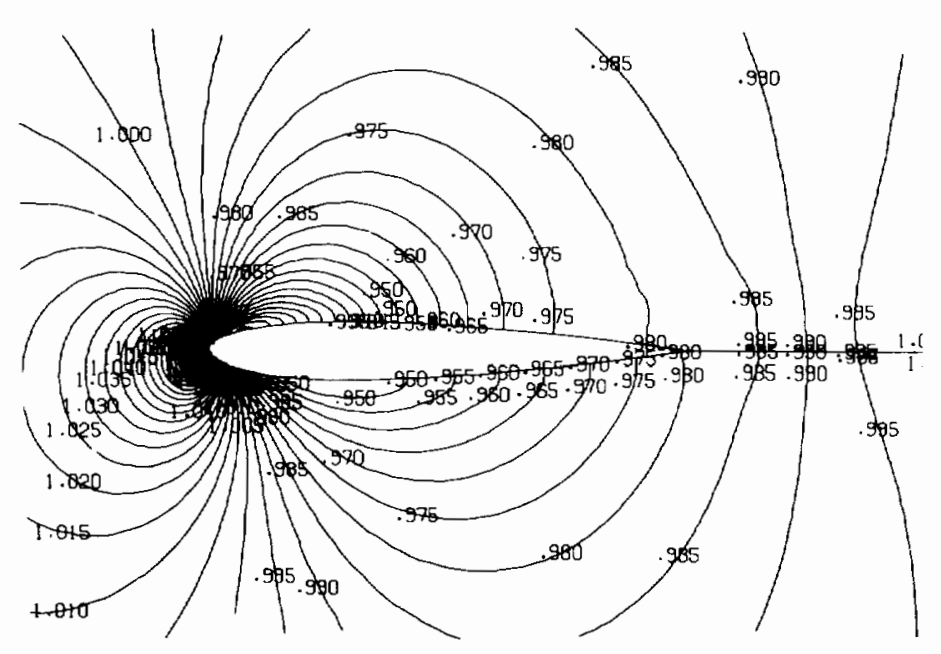

b) 5-wave model

Figure 12 NACA 0012, M $=0.5, \alpha=3^{\circ}, \operatorname{Re}=5000$, pressure contours, 2nd order accuracy 